\title{
VULNERACIÓN AL DERECHO A LA SALUD POR CONTAMINACIÓN DE PLOMO, EN VENTANILLA, 2017.
}

\section{VULNERATION OF THE RIGHT TO HEALTH BY LEAD CONTAMINATION, IN VENTANILLA, 2017.}

\author{
Karla FERNÁNDEZ MUNAYLLA \\ Dr. Jorge RODRIGUEZ FIGUEROA
}

\begin{abstract}
RESÚMEN
El presente artículo tiene por objetivo: Analizar cómo pese a existir normas legales aún se vulnera el Derecho a la Salud, por efecto de la Contaminación Ambiental por parte de empresas, que no tiene responsabilidad social, sus maquinarias emiten plomo en el Distrito de Ventanilla 2017. En tal sentido, en la presente investigación se ha evidenciado una amplia vulneración del derecho a la salud y medio ambiente producido por la contaminación ambiental por parte de las diversas empresas, las que emiten entre otros compuestos químicos tóxicos, dañinos a la salud, como es el plomo, lo que ha permitido comprobar mediante entrevistas y documentos oficiales, como se continua afectado, dañando, menoscabando, los derechos humanos a la vida, los derechos fundamentales donde, en primer orden afecta a los indefensos niños, ancianos y madres gestantes, quienes por vivir alrededor de dichas empresas industriales, siguen siendo los más vulnerables, centrándose en la desprotección por parte de las políticas del estado frente a la contaminación de compuestos químicos, siendo el plomo, el que más daño está causando a la salud.
\end{abstract}

Palabras claves: vulneración, derecho, Derecho a la Salud, Medio Ambiente y contaminación ambiental.

\begin{abstract}
The objective of this article is to: Analyze how, despite the existence of regulations, the Right to Health due to Environmental Pollution due to lead in the Ventanilla District 2017 is still violated. In this sense, it will be possible to demonstrate a broad development on the issue of the violation of the right to health and the environment produced by environmental contamination by lead as well as a small account on human rights and fundamental rights where children, the elderly and mothers Pregnant women are still the most vulnerable, focusing on the lack of protection against lead
\end{abstract}


IUS ET SCIENTIA (ISSN: 2444-8478) 2019, Vol.5, $\mathrm{n}^{\circ} 1, \mathrm{pp} .128-156$

VULNERACIÓN DEL DERECHO A LA SALUD POR CONTAMINACIÓN DE PLOMO EN VENTANILLA, 2017. Karla FERNÁNDEZ MUNAYLLA y Jorge RODRÍGUEZ FIGUEROA, jrodriguezfig@ gmail.com Universidad César Vallejo (Perú) Recibido: 05/02/2019. Aceptado: 16/05/2019 DOI: http://dx.doi.org/10.12795/IETSCIENTIA.2019.i01.06

contamination.

Keywords: violation, right, Right to Health, Environment and environmental pollution. 


\section{RESUMEN}

El presente artículo tiene por objetivos Determinar cómo se relacionan las normativas ambientales con el Derecho a la Salud, asimismo, identificar como la Contaminación Ambiental por plomo de las empresas metalúrgicas del Distrito de Ventanilla perjudican el bienestar de los pobladores Vulneración al Derecho a un Medio Ambiente que implica la prevención, orientación y valoración de los recursos ecológicos y la salud de los habitantes; en cuanto a la destrucción que ocasiona la Contaminación. Según el problema a plantear, la investigación solo se limitará al análisis que permita concluir con medidas efectivas que exijan el cumplimiento de las normas impuestas por el Estado, como en el caso del tema a investigar, la eliminación del accionante contaminante en la zona por el bienestar de la salud de los pobladores principalmente a niños y ancianos. En tal sentido, la presente investigación tiene como finalidad crear conciencia en las personas sobre sus posibles consecuencias si no se previene oportunamente la contaminación por plomo. La problemática en cuanto a Conciencia Ambiental y la de Ventanilla.

Cabe indicar que el presente artículo sigue las etapas que debe incluir el informe de un artículo científico son:

I. Introducción

II.Metodología

III. Resultados

IV. Discusión y conclusión

V. Bibliografía

A continuación se exponen cada una de las etapas antes mencionadas. 


\section{I.- INTRODUCCIÓN}

En la parte introductoria se presenta el problema del porqué de la investigación que en nuestro caso se ha tratado de la situación sobre la contaminación ambiental, por parte de empresas industriales, que sus equipos y maquinarias emiten compuestos químicos, entre los más alarmantes es el denominado plomo en el Distrito de Ventanilla.

Como lo muestra nuestra Constitución Política en su artículo $\mathrm{N}^{\circ} 1$, al señalar que toda persona tiene derecho al respeto de su dignidad, pues constituye el fin supremo del Estado y la sociedad. Sin embargo, no siempre es así, pues existen hechos que atentan contra persona como es, el debido desarrollo humano y su dignidad, preocupación del Estado que debe tomar con suma importancia el daño ambiental que afecta directamente a nuestro país, siendo considerado el segundo país a nivel mundial con mayor biodiversidad entre todos.

Es por ello que en esta parte introductoria se recogen los antecedentes del estudio, tanto a nivel nacional como internacional y que se precisan a continuación.

Asimismo, resulta relevante mencionar el aporte de nuestra Legislación Peruana en la Ley General de la Salud No 26842, (2004), referente a la salud, mediante el cual señala, que el Estado es el órgano protector encargado de salvaguardar, proteger y atender situaciones respecto a la salud de todas las personas, siendo también en el caso de personas con discapacidad física $o$ mental, entre los niños y adolescentes, y en aspectos generales a toda persona siendo aún esta misma de escasa solvencia económica o se encuentre en un abandono social.

Otro aporte resaltante es el de la Comision Interamericana de Derechos Humanos (1983), donde menciona que, el conservar una población y sus ciudadanos saludables era primordial contar con un medio ambiente saludable y apto para todos, es por ello que se llevó a cargo estudios para la eliminación de sustancias toxicas o desechos industriales ocasionadas por las empresas para disminuir el riesgo a enfermedades infeccionas y permanentes encontradas en el organismo.

Como antecedente nacional se cuenta con el aporte de La autora Alva, K. (2014). Fortalecimiento de la protección al ambiente y los recursos naturales en la Constitución Peruana de 1993. (Tesis para adquirir el Título Profesional de Abogada, Universidad Privada Antenor Orrego de Trujillo), donde concluye lo siguiente: "El aprovechamiento racional de los recursos naturales del país es parte de ese proceso de observar el derecho a un ambiente sano y equilibrado, sin perjudicar el bienestar y salud de las generaciones futuras $[\ldots]$ ".

Dicho Informe señala que los temas tratados respecto a la salud de una ciudad o una nación deben ser temas en primer asunto tanto a niveles nacionales como internacionales, conversadas y tratar de ser resueltas lo mejor y más pronto posible para que así se evite de cualquier manera, tomar medidas que no correspondan siendo evaluadas en realidades problemáticas parecidas a las 
mismas, porque son conflictos que pasan en todas partes del mundo, de distintas maneras y a diferente magnitud pero al final siempre terminan afectando el mismo punto que es el derecho a la salud.

Del mismo modo, mencionamos a Acosta (2011), indicando que la moralidad de cada ser hacia nuestro entorno y nuestra ayuda al planeta.

Como antecedente internacional se cuenta la Agencia de Protección Ambiental (2017). Hace mención en su portal virtual, "Qué se puede hacer para proteger a la familia contra la Intoxicación por Plomo", lo cual define al plomo como: "El plomo puede entrar en el ambiente de estos usos pasados y actuales, como también puede ser emitido en el ambiente de fuentes industriales y sitios contaminados, tales como fundidores de plomo anteriores.

Asimismo, resulta valioso el informe de la OEFA Nº 69/04, (2004), nos dice sobre la violación al derecho a la vida, la integridad personal, las garantías judiciales, y al desarrollo progresivo de los derechos económicos sociales y culturales todo ello, lleva a evaluar y recurrir al criterio medioambiental, así como la obligación del Estado peruano de responder ante lo acontecido.

Como se aprecia tanto a nivel nacional como internacional, la problemática ambiental, la fiscalización ambiental y la gestión de los residuos sólidos, ha sido una constante preocupación de los expertos y autores basado en el panorama que se tiene sobre un naturaleza tendría que ser protegida en cuanto a su vida, no solo por ser considerada en otros países ambientalistas como un sujeto de derecho, sino porque es lo afirmativo y correcto que se debe hacer; porque es la extensión de

la

ambiente equilibrado, limpio y habitable, que causa a posterior nuevos cambios, mejores beneficios, creando un mejor desarrollo de un ambiente limpio y habitable; de igual forma, fiscalizando que el daño sea controlado para que no siga perjudicando la vida de más personas, como consecuencia de un buen manejo y de las condiciones sanitarias necesarias que debemos tener en nuestro entorno.

\section{METODOLOGÍA}

Respecto a los aspectos metodológicos, mencionaremos los siguientes:

\section{a) Diseño}

En resumen, se aplicará el diseño de la teoría fundamentada, de Estrauus y Corbin (1990) debido a que las posibles conclusiones a las que se llegarán en una etapa de desarrollo estarán basadas en aquellos datos recogidos a lo largo de la investigación, así como información recogida de las teorías relacionadas al tema.

\section{b) Población y muestra}

Respecto a la población, debemos advertir que se trató de la población del distrito de Ventanilla y con una muestra de 10 operadores, conocedores y aplicadores de las normas administrativas sancionadoras de 
índole ambiental.

Nos referimos e modo particular a 7 especialistas profesionales en la rama de Derecho Constitucional y Derecho Ambiental así como también en profesionales de la Medicina.

Cabe indicar que para el muestreo se utilizó el procedimiento no aleatorio ya que los informantes claves sí fueron escogidos debido a

\section{c) Consideraciones éticas}

El presente artículo se llevó a cabo en cumplimiento con los parámetros establecidos por la Universidad Cesar Vallejo, encontrándose en el marco cualitativo, considerando las indicaciones señaladas en la normativa. Asimismo se respetó los derechos de autor de los textos consultados y de la mención de la identidad de los informantes claves. Para el citado de las fuentes se aplicó las normas de estilo de la Asociación de Psicología Americana, lo que asegura el pleno respeto con los principios de derecho de autor, lo cual proporciona una fuente confiable para sustentar bases sólidas en el desarrollo del artículo.

\section{RESULTADOS}

\section{a) Recolección de los datos}

En palabras de Baptista, Fernández y Hernández (2014), la recolección de datos resulta medular, puesto que es lo Descripción de resultados de la técnica de Análisis de fuente documental que se refiere a un estudio cualitativo ya que se busca obtener datos, ya que los datos que importan sus conocimientos, capacidades y manejo teórico y práctico del tema, como corresponde a un artículo de investigación.

Por lo expuesto se ha logrado que la población y la muestra escogida es representativa, significativa y apropiada para alcanzar los Resultados de investigación esperados.

son los conceptos, pensamientos, percepciones, siendo que se recogen para luego analizarlos y comprenderlos (p.397). Igualmente Huamanchumo y Rodriguez, señalan que los Resultados es el punto mas importante en una investigación, por su originalidad.

Al respecto cabe indicar que la recolección de los datos estuvo relacionado con las técnicas de recolección de datos aplicadas. Nos referimos a la entrevista, análisis de fuente documental $y$ encuesta poblacional aplicada. Todas estas técnicas nos han permitido obtener datos variados, diversos, integrales y válidos, toda vez que se trató de obtener datos que nos ayuden a confirmar los Supuestos Jurídicos y obtener Resultados conforme a los Objetivos de la investigación planteada.

\section{b) Análisis de los datos}

Luego de la recolección de los datos, era importante precisar el modo en que estos se iban a analizar. Para el presente artículo de investigación se enfocó en la interpretación en base a la actividad que los investigadores cualitativos realizan, donde se hacen registros descriptivos de los sucesos y/o 
fenómenos que se centran en el estudio, como la observación, entrevistas no estructuradas.

También ha ayudado para el análisis de los datos el enfoque hermenéutico de las normas relacionadas al derecho a un ambiente sano y a la gestión de los residuos sólidos

\section{c) Presentación de los datos}

A continuación se precisan los resultados obtenidos en el presente estudio.

1. Descripción de resultados de la técnica de Análisis de fuente documental

Respecto, al objetivo general: Analizar cómo pese a existir normativa aún se vulnere el Derecho a la Salud por Contaminación Ambiental por plomo en el Distrito de Ventanilla 2017. Se ha analizado el siguiente documento:

a) Sentencia recaída en el 


\section{Expediente $\quad \mathbf{N}^{\circ} \quad$ 009-2017- OEFA/TFA-SEPIM}

En el presente documento materia de análisis para justificar el objetivo general, fue la sentencia del Expediente $\quad \mathrm{N}^{\circ} \quad$ 009-2017OEFA/TFA-SEPIM, proceso interpuesto por La Dirección de Supervisión Ambiental sobre la empresa Metalexacto S.R.L, respectivamente dentro de los considerandos y fundamentos expuestos en la referida se fundamenta la vulneración del Derecho a la Salud por Contaminación Ambiental por plomo en el Distrito de Ventanilla, aduciendo que la falta de control ambiental es el problema central en cuanto a fiscalización, por lo siguiente que señala " La cuestión en el presente caso versa sobre el dictado de una medida preventiva por parte de la Dirección de Supervisión consistente en el cese inmediato de toda forma de emisiones producto de sus actividades en la Planta Ventanilla mientras no acredite ante la Dirección de Supervisión la actualización del instrumento de gestión ambiental aprobado por la autoridad competente conforme a Ley", siendo así el resultado; Dirección Regional de Salud, entre las fechas del 20 de setiembre y el 6 de octubre del 2014, en su último monitoreo confirmó la presencia del agente contaminante en la zona, el cual en su estudio determinó que las cantidades de plomo recogidas son 1,55 microgramos por metro cúbico y por el cadmio son 0,32 microgramos por metro cúbico, de modo que en el estudio sobrepasaban los máximos registros permitidos, Asimismo, los límites establecidos por la Organización Mundial de la Salud en el componente de plomo son de 0,5 microgramos por metro cúbico y 0,25 microgramos por metro cúbico para cadmio, razón suficiente para dar una idea a la magnitud del problema, en relación a este primer estudio, se dio en evidencia los efectos del plomo en la salud física y psicológica de los evaluados y la reducción de peso y talla que afectan gravemente la salud de los pobladores, quedando ratificado el mencionado objetivo general.

Respecto, al objetivo específico 1: Determinar cómo se relacionan las normativas ambientales con el Derecho a la salud. Se ha analizado los siguientes documentos:

\section{a) b) Sentencia recaída en el} Expediente $\mathrm{N}^{\circ}$ 2002-2006-PC/TC.

, establece en el fallo que Declarar FUNDADA en parte la demanda de cumplimiento presentada por Pablo Miguel, ordena que el Ministerio de Salud, en el plazo de treinta (30) días, implemente un sistema de emergencia para atender la salud de la personas contaminadas por plomo en la ciudad de La Oroya, debiendo priorizar la atención médica especializada de niños y mujeres gestantes, a efectos de su inmediata recuperación, conforme se expone en los fundamentos 59 a 61 de la presente sentencia, bajo apercibimiento de aplicarse a los responsables las medidas coercitivas establecidas en el Código Procesal Constitucional.

Se cumpla con realizar todas aquellas acciones a la expedición del diagnóstico de línea base, conforme lo prescribe el artículo $11^{\circ}$ del Decreto Supremo 074-2001-PCM, Reglamento de Estándares Nacionales de Calidad Ambiental del Aire, de modo tal que, cuanto antes, puedan implementarse los respectivos planes de acción para el mejoramiento de la calidad del aire en la ciudad de La Oroya y el artículo 105 de la Ley 26842.

Finalmente, con ello se comprueba que los estándares de calidad dañados por la Contaminación Ambiental por plomo es causal de 
preocupación y atención conjuntamente con el accionar de las autoridades competentes que no toman las medidas adecuadas para dejar de contaminar y de controlar la contaminación ya encontrada, con ello el Tribunal Constitucional como en el caso presentado llego a términos donde no solo se basaban en legislación nacional sino interpretaciones internacionales que avalan nuestros actos y el hecho de contaminación por plomo presente en nuestro país.

\section{c) Informe $\mathrm{N}^{\circ} \mathbf{6 9 / 0 4}$}

El documento materia de análisis para justificar el objetivo específico 2, fue el Informe $\mathrm{N}^{\circ}$ 69/04 realizado por la Comisión Interamericana de Derechos Humanos, proceso de peticiónadmisibilidad interpuesto por la Coordinadora Nacional de Comunidades del Perú Afectadas por la Minería, CONACAMI en la cual se alega la responsabilidad de la República del Perú por la violación de los derechos fundamentales, individuales y colectivos de los miembros de la Comunidad de San Mateo de Huanchor, donde los pobladores sufren contaminación ambiental ocasionados por la empresa minera Mayoc en una cancha de relaves; tal como señala: "Los efectos de los contaminantes ubicados en la cancha de relave Mayoc han ocasionado una crisis en la salud pública de la población de San Mateo de Huanchor, donde los más afectados son los niños de la población quienes cuentan con altos índices de plomo, arsénico y mercurio causando daños irreparables en el organismo", siendo así el resultado que se obtuvo de acuerdo al mencionado objetivo específico 2 .

Finalmente, la Comisión considera que los recursos interpuestos ante la autoridad administrativa y judicial con el objeto de buscar la tutela jurídica de los derechos afectados en perjuicio de los pobladores de San Mateo de Huanchor han resultado ineficaces, como es el presente caso se le otorgo su petición Declarar admisible el presente caso con relación a la presunta violación al derecho a la vida, la integridad personal, las garantías judiciales, la protección a la familia, del niño, la propiedad, protección judicial y al desarrollo progresivo de los derechos económicos sociales y culturales establecidos en los artículos 4, 5, 8, 17, 19, 21, 25, 26, de la Convención Americana, en concordancia con los artículos 1(1) y 2 del mismo instrumento, en esta materia, de derechos fundamentales.

\section{Descripción de resultados de la técnica: Entrevista}

Según Bernal (2016, p. 10) la descripción de resultados debe realizarse con la finalidad de interpretar los resultados obtenidos de la aplicación del instrumento, esto siempre concordante con el problema de investigación, los objetivos propuestos y los supuestos planteados. Asimismo de acuerdo con las teorías desarrolladas en el marco teórico, lo que se debe tomar en cuenta es que se cumpla con evaluar si los resultados del estudio confirman las teorías, o no las confirman o que generan debates con la teoría ya existente.

En ese sentido seguidamente, se describe y analiza los resultados obtenidos de la información de las entrevistas realizadas comprendidas durante los meses de setiembre a diciembre del presente año, mencionando que la 
información obtenida constituye la fuente principal para demostrar los supuestos jurídicos especifico de esta tesis, partiendo de que cada objetivo consta de 2 preguntas en su haber, siendo un total de 6 preguntas, según como se detalla a continuación:

\section{OBJETIVO GENERAL:}

Analizar cómo pese a existir normativa aún se vulnere el Derecho a la Salud por Contaminación Ambiental por plomo en el Distrito de Ventanilla.

De acuerdo al enunciado número 1.- Explique Usted. ¿Cómo pese a existir normativa aún se vulnere el Derecho a la Salud por Contaminación Ambiental por plomo en el Distrito de Ventanilla?

a) Los entrevistados Santisteban (2018), Troncos (2018), La Torre (2018), Inga (2018), Díaz (2018), Meléndez (2018), Huamani (2018), consideran que si se vulnera el Derecho a la Salud por Contaminación Ambiental por plomo, puesto que no existe un control adecuado a cargo de los órganos fiscalizadores que deberían de proteger y velar por nuestros derechos y mucho menos existe apoyo por parte de la Región Callao.

Al respecto, Santisteban (2018) indica que "los estándares de calidad y sanidad en el Distrito de Ventanilla no son los adecuados para toda la población por igual ya que la contaminación afecta en mayor magnitud a ciertas zonas del Distrito más que a otras", Troncos (2018) señala que "la vulneración del Derecho a la Salud por Contaminación Ambiental por plomo sigue siendo latente por la falta de fiscalización de la Dirección de Gestión AmbientalCallao, ente fiscalizador de los parámetros y estándares del medio ambiente del Distrito".

Asimismo, la Dra. La Torre (2018) dispone que "Estos derechos están siendo vulnerados a pesar de encontrarse regulados en la Constitución política del Perú, determinando en su art. 2 inciso 22, su importancia respecto a la salud de los pobladores de Ventanilla".

Inga (2018) al respecto menciona que "Se seguirá vulnerando los Derechos tanto a la salud como al medio ambiente porque la Municipalidad Distrital de Ventanilla conjuntamente con la Región Callao no tomar accionar ni mucho menos presentan sus quejas ante el Ministerio de la Producción y el Ministerio de la Salud dando a conocer que los hechos son materia de investigación y de extremo peligro para todos".

Leyva (2018) dispone que "Se seguirá vulnerando los Derechos como a la salud y al medio ambiente hasta que no exista una correcta fiscalización del accionar de las empresas y un control interno mejorado por parte de la Municipalidad de Ventanilla que se preocupe por el bienestar de su gente".

Meléndez (2018) manifiesta que "Si bien a pesar de existir normativa aún se vulnere el Derecho a la Salud por Contaminación Ambiental por plomo es responsabilidad del Estado y quien se desprende de los mismos para que hagan efectivas las actuaciones respecto a contaminación ambiental y el estado crítico de la población".

Asimismo, Huamani

sostuvo que "La vulneración al Derecho a la Salud por Contaminación Ambiental por plomo es por la falta de control de 
la aplicación de las normativas a la realidad y que estas mismas deben de desprenderse de las normativas y políticas ambientales correspondientes"

b) Los entrevistados Castro (2018), Meza (2018) y Díaz (2018) manifiestan que "Haciendo precisión en los términos de calidad y equilibrio en el ambiente que nos rodea, estipulado en el Ley General del Ambiente $\mathrm{N}^{\circ}$ 28611 donde se determinan normativas que protegen tanto al ambiente como al ciudadano pidiendo apoyo también por parte del Ministerio del Ambiente, que su vulneración se debe a que no se encuentra debidamente derivada estas funciones como es el caso del Ministerio de la Producción, OEFA y la Municipalidad Distrital de Ventanilla teniendo acciones limitadas por parte de las empresas contaminadoras que son netamente competencia de OEFA, esta delegación de funciones crea confusión y poca acción por parte de quien en verdad quiere trabajar por la salud de los pobladores, haciendo hincapié en que también se vulnera otros derechos como el Derecho a la Vida".

En efecto, respecto a la primera pregunta se ha podido señalar que de los 10 entrevistados, $\underline{7}$ coinciden en que si existe una vulneración del Derecho a la Salud por Contaminación Ambiental en el Distrito de Ventanilla pese a existir norma que lo regula y que de ello se determina que no se haga efectiva la adecuada protección a nuestros derechos, regulado en la Constitución Política del Perú, 3 entrevistados señalan que no hay un adecuado tratamiento respecto a la competencia y derivación de funciones para cada entidad por la ineficiencia de nuestro sistema jurídico pese a que tenemos leyes como la Ley General de la Salud $\mathrm{N}^{\circ} 26842$ y la Ley General del Ambiente $\mathrm{N}^{\circ} 28611$ que los protegen también porque de ello se desprende la idea que no solo involucra estos derechos mencionados anteriormente sino que tienen que ser de estricto cumplimiento para todos tanto para personas jurídicas o privadas. De acuerdo a la pregunta 2, En su opinión, ¿Cómo se debe prevenir la Contaminación Ambiental por plomo en el Distrito de Ventanilla?, se ha obtenido los siguientes resultados:

a) Los entrevistados Santisteban (2018), Troncos (2018), La Torre (2018), Meza (2018), Meléndez (2018), Huamani (2018), Castro (2018), Leyva (2018), Inga (2018) consideran que la forma de prevenir la Contaminación Ambiental por plomo en el Distrito de Ventanilla es dejar tener un mejor control de las empresas que van a contaminar y depurar a las empresas contaminadoras de la zona.

Al respecto, Santisteban (2018) señala que "la mejor manera para prevenir la Contaminación Ambiental por plomo es tener un estricto control basado en la Ley General del Ambiente $N^{\circ} 28611$ y al mismo tiempo tratar de reposicionar a los pobladores de los alrededores a zonas menos contaminadas.

Troncos (2018) manifiesta que “ Cuando el control interno sea más eficiente podremos hablar en cuanto a sanciones y restricciones amparado bajo La Constitución política del Perú y nuestros tratados con la Comisión Interamericana de Derechos 


\section{Humanos".}

Meza (2018) menciona que "Se debe dejar de contaminar y centrar las ideas en un ambiente sano para la crianza de los niños y madres gestantes al mismo tiempo de la salud y vida de cada poblador como lo estipula la Ley General de la Salud $26842^{\circ}$ donde en su artículo $103^{\circ}$ menciona la obligación del estado al otorgar y brindar atención y protección médica a todos", Meléndez (2018) señala que "La mejor prevención de la Contaminación Ambiental por plomo en el Distrito de Ventanilla es mejorar los estándares de calidad y límites permisibles establecidos en la Ley General del Ambiente 28611 ${ }^{\circ}$ regulados para poner un equilibro y balance respecto a contaminación y su respeto a la calidad de vida".

Huamani (2018) aduce que "Para prevenir la Contaminación Ambiental por plomo se debe de hacer una nueva investigación y evaluación por parte de la OEFA ente encargado de investigar casos en materia de gestión ambiental y reevaluar los índices de contaminación en la zona y de ser concerniente evacuar la zona afectada y esterilizar el ambiente contaminado", Castro (2018) menciona que "Una de las maneras para prevenir la Contaminación Ambiental por plomo en el Distrito de Ventanilla es clausurar todas aquellas empresas metalúrgicas que excedan los alcances ambientales o que no sean formalizadas bajo el régimen empresarial y con autorización de la Municipalidad Distrital de Ventanilla para dejar de contaminar así más zonas y combatir contra un mal que causa daños permanentes en la salud física y mental de las personas.

b) El entrevistado Díaz (2018) señala que desde el punto de vista médico " una de las formas de prevenir la Contaminación Ambiental es que se designen zonas especializadas y libres de contaminantes para la población de Ventanilla, y que es parcialmente aceptado por el estado ya que ventanilla al ser un distrito industrial, por ende se procedería a mejorar su calidad y disminuir su productividad caso que el estado no estaría de acuerdo porque no considera temas de vital importancia como el derecho a la salud.

En consecuencia, respecto a la segunda pregunta, se ha podido determinar que de los 10 entrevistados, 9 coinciden que la mejor forma para prevenir la Contaminación es no pasar por alto aquellas empresas informales y ser más estrictos con aquellas empresas que se dedican al mismo rubro, los cuales determinan según la Constitución Política del Perú las normativas respecto a las obligaciones de prestar atención y un medio ambiente sano para las futuras generaciones basado en las investigaciones de la OEFA en el 2016, y siendo entre ellos, 1 entrevistado que establece que la designación de zonas industriales debe de estar separada de la población urbana del distrito de Ventanilla otorgándole asimismo valor por la dignidad de la persona siendo una norma y un valor estipulada en el artículo $1^{\circ}$ de la Constitución Política del Perú. OBJETIVO ESPECÍFICO 1:

Determinar cómo se relacionan las normativas ambientales con el Derecho a la salud.

De acuerdo a la pregunta número 1 de mi objetivo específico 1 : 
1. Señale Usted, ¿En qué consiste la relación de la normativa ambiental con el Derecho a la Salud?, se ha obtenido el siguiente resultado:

a) De los entrevistados, Santisteban (2018), Troncos (2018), La Torre (2018), Castro (2018), Díaz (2018), Meléndez (2018), Huamani (2018), Leyva (2018), Meza (2018) concuerdan en que si existe una relación entre normativa y la realidad aplicada al Distrito de Ventanilla, donde la problemática versa en tener un ambiente sano y como consecuencia afectaría nuestra salud según las normativas vigentes.

Al respecto, Santisteban (2018) manifiesta que "La relación que existe entre la normativa Ambiental con el Derecho a la Salud se basa en el impacto constitucional que se desprende no solo de estos derechos sino que de igual forma afectarían otros derechos como la dignidad, el derecho a la vida, entre otros"; asimismo, Troncos (2018) señala que " la relación es muy estrecha porque la salud es un derecho fundamental de la persona y el derecho como tal esta para defender ese derecho fundamental, tanto de nuestro entorno como de nuestra salud regulados ambos en la Constitución Política del Perú y en sus legislaciones pertinentes".

La Torre (2018) menciona que "si existe una relación siendo consecuentes una con la otra, si no se tiene un ambiente adecuado y equilibrado se verá afectado nuestro derecho al ambiente, ello mismo conlleva a que nuestra salud sea afectada y por ende pondría en riesgo al mismo tiempo nuestra salud, como consecuencias se presentaría problemas de salud a causa de las empresas que contaminan nuestro ambiente".

Asimismo, Meléndez (2018), menciona que " Claro que existe una relación tanto con la comunidad en cuanto a gestión ambiental y con las empresas que quieren destacar en lo industrial, así como las normas están conectadas de igual forma las consecuencias de las mismas repercutan en la sociedad" de igual forma, Huamani (2018) señala que “ $\mathrm{Si}$ existe una relación interdependiente quiere decir, que no se puede cumplir solo una, sino que todos estos derechos están conectados tanto para buscar el bienestar de las personas personalmente y del Distrito asegurando con ellas la salud pública de todos".

El entrevistado Inga (2018) señala que "la relación que guardan serían aplicados en forma equitativa, quiere decir, que si efectivamente tienen relación con la problemática de contaminación por plomo pero que al mismo tiempo tienen que ver los límites permisibles reconocidos por el Ministerio de la Producción en cuanto a sanidad respecta de nuestro Distrito y reevaluar si se produce como causa o efecto o acción y reacción al problema de investigación".

En consecuencia, respecto a la primera pregunta de mi objetivo específico 1, se ha podido señalar que de los 10 entrevistados, $\underline{\operatorname{los} 10}$ están de acuerdo que las normativas ambientales con el Derecho a la Salud se encuentran estrechamente correlacionadas una con la otra, de modo que, los especialistas aducen que si bien la normativa tanto como la política ambiental y los estándares de 
sanidad que se deben de tener para la vida y la buena salud tienen relación, poniendo como ejemplo que si no vivimos en un lugar con un medio ambiente equilibrado nos produciría enfermedades, infecciones entre otros, por ende se afectaría nuestro Derecho a la Salud en temas normativos se encuentran relacionados y en los ámbitos aplicados a la vida real y la sociedad en común.

La relación de la normativa ambiental como en la expectativa con la realidad problemática en este caso la Contaminación Ambiental por plomo, es un ejemplo aplicado a la realidad, en conclusión, tiene una relación tanto normativa, social y problemática que abarcan distintos ámbitos pero que siempre se relacionan.

De acuerdo a la pregunta número 2 de mi objetivo específico 1.Explique Usted, ¿Por qué motivo no se cumple con el Derecho a la Salud conforme lo señala la Constitución? , se ha obtenido el siguiente resultado:

b) Los entrevistados Santisteban (2018), Díaz (2018), La Torre (2018), Castro (2018), Díaz (2018), Meléndez (2018), Huamani (2018), consideran que no se cumple con el Derecho a la Salud conforme lo señala la Constitución por falta de control y fiscalización por parte de la Municipalidad Distrital de Ventanilla y en todo caso de la Región Callao con respecto a la Contaminación Ambiental por plomo en el Distrito de Ventanilla. Según Santisteban (2018) manifiesta que el Derecho a la salud no se cumple porque no se le da la debida importancia y concientización a los temas ambientales al ser estos menos valorizados por el mismo ser humano y propiamente el estado", Díaz (2018) señala que "Los temas de la salud tienen menos valor que el crecimiento de un Distrito porque prefieren contribuir al desarrollo de un estado olvidando los límites permisibles de vivir en un medio ambiente sano", La Torre (2018) menciona que "el valor que el estado está dándole al medio ambiente y la salud tiene que ser por parte de las autoridades correspondientes que están mayormente más centradas en otros temas que en los que son realmente importantes para el desarrollo de la vida".

$\mathrm{Al}$ respecto de la segunda pregunta del objetivo específico 1 , se ha podido señala que de los 10 entrevistados, 7 de las personas señalan que es por la falta de concientización y sensibilidad $\underline{\text { social que vienen por parte de las }}$ autoridades hacia nuestro ambiente natural, porque ellos aducen que la ley existe en la Constitución Política del Perú art. $7^{\circ}$, pero no hay una política de gobierno que exija o que trabaje conjuntamente con el Distrito de Ventanilla para ver una mejoría en cuanto a la salud y el bienestar social de todos, 3 de los entrevistados están de acuerdo que el apoyo que se le da a otras situaciones que acontece el Distrito tanto como el desarrollo económico son más importantes y no ponen en riesgo a todos los pobladores solo por un beneficio monetario, el valor del Derecho a la Salud como pilar básico para el desarrollo de una población sana, no solo debe de basarse en tener la normativa para poder aplicarla sino ponerla en práctica ya que las normas se crearon para aplicarlas y no solo para redactarlas según como lo regula la Constitución Política del Perú y La Ley General 
de Salud $\mathrm{N}^{\circ} 26842$.

\section{OBJETIVO ESPECÍFICO 2}

Identificar como la

Contaminación Ambiental por plomo de las Empresas Metalúrgicas del Distrito de Ventanilla perjudica el bienestar de los pobladores principalmente a niños y ancianos.

De acuerdo a la pregunta número 1 del objetivo específico 2, Precise Usted, ¿Cuál es la forma más frecuente de contaminación que producen las Empresas Metalúrgicas del Distrito de Ventanilla que perjudiquen el bienestar de los pobladores principalmente a niños y ancianos?

a) Los entrevistados Santisteban (2018), Díaz (2018), La Torre (2018), Castro (2018), Leyva (2018), Meléndez (2018), Troncos (2018), consideran que la forma más frecuente de Contaminación que producen las empresas metalúrgicas es por vía del aire, donde los gases y combustibles mediante el humo son absorbidos por el ambiente perjudicando el bienestar principalmente de los niños y ancianos.

Según Santisteban (2018) señala que "efectivamente las personas más vulnerables son los niños y los ancianos quienes tienen niveles de defensas en pleno desarrollo o mínimas respectivamente, con los humos que producen las maquinas son los más perjudiciales y enviados al ambiente nosotros los absorbemos y no solo dañando la capa de ozono sino nuestra salud".

Díaz (2018) indica que "Los niveles de plomo alcanzados en el Distrito de Ventanilla producido por las empresas metalúrgicas según los estándares de calidad del aire en Ventanilla no son los adecuados y si, efectivamente dañan no solo la capa de ozono, la naturaleza y a todos", la Dra. La Torre (2018) menciona que "La gran parte de contaminación que se produce por parte de las empresas metalúrgicas en base a su estructura y construcción es por sus humos, donde claramente nosotros los respiramos, inhalamos y ciertamente nos contaminamos".

Meléndrez (2018) señala que "la manera más frecuente de contaminación producida por las empresas metalúrgicas es por el aire, cuando las empresas a través de sus máquinas desprenden sus humos al cielo, contaminan el ambiente y el aire que diariamente respiramos esto hace que nosotros mismos inhalemos esos gases tóxicos que perjudican nuestro cuerpo y con mayor magnitud a los niño y ancianos".

Troncos (2018) menciona que "Las empresas metalúrgicas encargadas de procesar el plomo contaminan de diferentes maneras pero el mayor daño lo hacen en base a los humos que emiten sus maquinarias, porque la población respira esos humos y daña nuestro sistema, impidiendo que los niños respiren con normalidad asimismo, los adultos sufran de problemas respiratorios también, igual son los más vulnerables conjuntamente con las madres gestantes que esperan a un ser humano en formación, causándole que puedan tener problemas desde el tiempo en formación".

En consecuencia, respecto a la primera pregunta de mi objetivo específico 2 , se ha podido señalar que de los 10 entrevistados, $\underline{\operatorname{los} 10}$ concuerdan en que la principal problemática de contaminación 
que es producida por las empresas metalúrgicas es por el aire, cuando las maquinarias como los materiales químicos que se emplean y el no control de riesgos de contaminación que producen cuando se instalan en un determinado lugar del Distrito, como consecuencia, se produce la contaminación por parte de las empresas en materia de suelo y aire que contamina el ambiente; además el especialista medico en la materia opina que los niveles más altos de contaminación son a niveles inhalatorios, ya que no es necesario que las empresas emitan vapores con plomo para contaminar sino que la simple instalación y cercanía con el elemento químico daña a través de las corrientes de vientos naturales que pasan por el Distrito que van justamente hacia las zonas rurales donde se encuentran viviendo niños, ancianos y también madres gestantes, contaminando no solo por plomo sino también por cadmio tal y como lo determinan los estudios ambientales realizados en el lugar y no cumpliendo con la Ley General del Ambiente $\mathrm{N}^{\circ}$ 28611.

De acuerdo a la pregunta número 2 del objetivo específico 2, Desde el punto de vista jurídico/ médico, ¿Qué efectos produce la Contaminación Ambiental por plomo en los niños y ancianos?, se ha obtenido el siguiente resultado:

b) Los entrevistados Santisteban (2018), Inga (2018), La Torre (2018), Meza (2018), Huamani (2018), Meléndez (2018), Troncos (2018), consideran que los efectos jurídicos que produce la Contaminación ambiental por plomo en los niños y ancianos es la afectación a sus derechos fundamentales estipulada en la Constitución Política del Perú, llevando así un proceso por las vías legales como la vulneración a sus derechos tales como el Derecho a la vida, la Salud, el ambiente entre otros.

Al respecto, Santisteban (2018) indica que "los efectos jurídicos que produce la Contaminación Ambiental por plomo es la contravención a la norma constitucional, indicando que estos derechos fundamentales para la existencia del ser humano son vulnerados y afectados en su totalidad causando que recurran a una demanda por la via civil como una acción de amparo para que se cumpla con sus derechos que son actualmente vulnerados, en tal caso los representantes legales de los niños podrían interponer una demanda por la vulneración de sus derechos".

Igualmente, Troncos (2018) manifiesta que "Los efectos jurídicos que tiene la Contaminación Ambiental por plomo que afecta en mayor magnitud a los niños y adolescentes son el daño a sus derechos fundamentales, estipulados en la Constitución Política del Perú del cual se desprenden como ley de mayor y máxima jerarquía en nuestra legislación peruana logrando así que si no podríamos acudir o tomar una buna decisión por la legislación peruana podamos acudir a los tratados internacionales como en el caso de las minerías".

En consecuencia, en relación a la segunda pregunta del objetivo específico dos, se ha podido verificar que de los 10 entrevistados, 7 de los especialistas 
jurídicos mencionaron que al encontrarse contaminación en el lugar, estarían afectando tanto sus derechos, como el Derecho a la Dignidad, el Derecho a la Vida, el Derecho a un Medio Ambiente sano y el Derecho a la Salud como derechos fundamentales que rigen a nuestra Legislación Peruana, contemplados dentro de la Constitución Política del Perú, frente a ello se podría acudir a las denuncias correspondientes en el Ministerio del Ambiente, actuando de forma específica primero por la vía civil con una demanda ejerciendo su derecho de acción y el temas penales como los delitos contra la vida, el ambiente y la salud, correspondientes al caso, ahora bien en cuanto a los 3 especialistas médicos estuvieron de acuerdo en que los niños y adultos son los más propensos a desarrollar enfermedades producto al contacto continuo y diario al plomo produciendo anemia hemolítica y crónica, donde $\underline{2}$ entrevistados señalan que respecto al contacto diario con el plomo tiene como consecuencia daños permanentes que son irreversibles para el desarrollo de una vida normal y cotidiana, el desgaste de los pulmones, plomo en sangre, todos estos son causales de riesgo para la vida de una persona y que tienen que ser combatidos de una vez por todas, por otro lado, 1entrevistado alega que no solo afectaría directamente la salud de los más vulnerables en este caso niños y ancianos sino menciono la inclusión de las madres gestantes y las malformaciones que podrían ocasionarse en el feto por el plomo donde se vulneran directamente nuestro Derecho a la Salud y Medioambiente, regulados respectivamente en La Ley General de la Salud y la Ley
General del Ambiente.

Al respecto, cabe analizar que si efectivamente a pesar de encontrarse normativa tanto Constitucional y precedentes que traten sobre temas de gestión ambiental y salud aún se vean vulnerados nuestros derechos y de acuerdo a las entrevistas realizadas a los especialistas tanto en materia constitucional como ambiental y asimismo en los informes médicos por lo que se desglosara de cada uno de las entrevistas, y se dará una mejor interpretación y análisis a las respuestas de los entrevistados.

\section{Descripción de resultados del análisis de la técnica de cuestionario}

\section{Análisis del cuestionario}

El cuestionario nos ayudó a reconocer las concepciones que tienen los pobladores del Distrito de Ventanilla respecto a este tema de investigación, es por ello que dicho cuestionario está dirigido a ellos. Fueron 10 personas encuestadas, entre las edades de 20 y 55 años, el presente cuestionario se llevó a cabo el día 23 de noviembre del presente año.

OBJETIVO GENERAL:

Analizar cómo pese a existir normativa aún se vulnere el Derecho a la Salud por Contaminación Ambiental por plomo en el Distrito de Ventanilla 2017.

1. ¿Considera usted que pese a existir normativa ambiental aún se siga vulnerando el Derecho a la Salud por Contaminación Ambiental por plomo en el Distrito de Ventanilla? Del $100 \%$ de los encuestados, el $100 \%$ asegura que la norma existe pero que aún se vulnera sus 
derechos y por lo tanto sigue habiendo Contaminación Ambiental por plomo en el Distrito.

\section{PREGUNTA N¹}

$$
\square \mathrm{SI} \quad \mathrm{NO}
$$

$100 \%$

\section{: Elaboración propia}

Del grafico anterior, podemos observar que el $100 \%$ de las personas afirma que se vulnera sus derechos a pesar que existe una normativa que la regula y como en el caso presentado es la vulneración del derecho a la salud por Contaminación Ambiental por plomo.

2. ¿Conoce usted Centros de Atención a la Salud en el Distrito de Ventanilla que den información sobre la contaminación del ambiente?

Del $100 \%$ de los encuestados, el $40 \%$ conoce centros de salud que den información sobre contaminación en el Distrito de Ventanilla y el otro $60 \%$ desconoce que se brinde información respecto a Contaminación Ambiental por plomo.

PREGUNTA N²

$\square \mathrm{SI} \quad \mathrm{NO}$

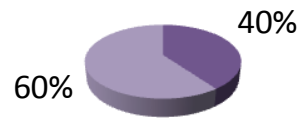

Fuente: Elaboración propia

Del grafico anterior, podemos observar que el $60 \%$ de la población no tiene conocimiento que en los centros de salud médica del Distrito se brinda información sobre contaminación ambiental siendo un tema latente.

3. ¿Tiene conocimiento del Daño que puede ocasionar la Contaminación del plomo en el cuerpo de una persona?

Del $100 \%$ de los encuestados, el $80 \%$ conoce los síntomas y daños que produce en su cuerpo el plomo y los problemas permanentes que ocasionan en su salud y el $20 \%$ no tiene conocimiento de los daños que puede ocasionar la Contaminación Ambiental del plomo.

PREGUNTA $\mathbf{N}^{\circ} 3$

$$
\square \mathrm{SI} \quad \mathrm{NO}
$$

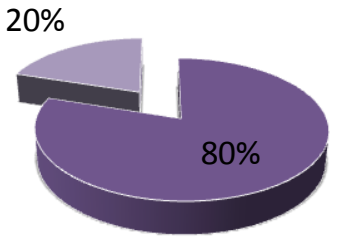

Fuente: Elaboración propia

Del gráfico anterior, podemos observar que el $80 \%$ de las personas tiene conocimiento de los daños que puede ocasionar en su cuerpo la contaminación ambiental por plomo.

4. ¿Considera usted que las autoridades de salud respetan el Derecho a un medio ambiente sano y limpio en su Distrito? Del 100\% de los encuestados, el $90 \%$ no considera que las autoridades de salud respeten su Derecho a la Salud y a un Medio Ambiente sano porque aseguran que sigue habiendo contaminación en el Distrito mientras que el $10 \%$ considera que si se respeta el 
Derecho a un medio ambiente sano y limpio en su Distrito.

\section{PREGUNTA N 4 sI}

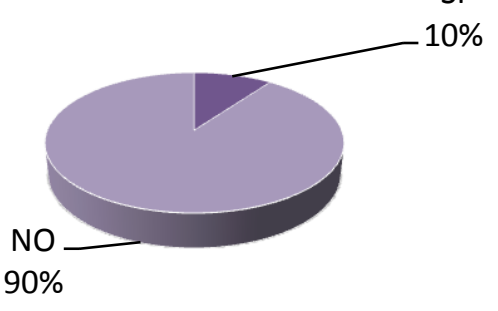

Fuente: Elaboración propia

Del grafico anterior, podemos observar que el $90 \%$ considera que las autoridades de salud respetan el Derecho a un medio ambiente sano y limpio en su Distrito.

5. ¿Está de acuerdo con las acciones sanitarias que se toman en su Distrito para brindarle una mejor vivencia y cuidar su salud?

Del $100 \%$ de los encuestados, el $60 \%$ está de acuerdo y considera que el Distrito ha mejorado en cuanto a medidas sanitarias respecta, mejorando la calidad de vida de sus hijos y su salud y el $40 \%$ no están de acuerdo de las acciones sanitarias que se toman en su Distrito.

\section{PREGUNTA $N^{\circ} 5$}

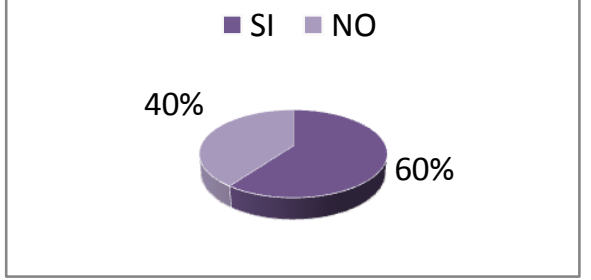

Fuente: Elaboración propia

Del grafico anterior, el $60 \%$ de los pobladores está de acuerdo con que las acciones sanitarias del Distrito de Ventanilla brindan una mejor vivencia y cuidan su salud.

6. ¿Considera que el Derecho a la Salud está relacionado con el Derecho a un Medio Ambiente sano?

Del $100 \%$ de los encuestados, el $100 \%$ de los pobladores están totalmente de acuerdo con que el Derecho a la Salud y el Medio Ambiente son derechos que tienen una gran relación entre sí.

\section{PREGUNTA N ${ }^{\circ} 6$}

$$
\square \mathrm{SI} \text { NO }
$$

$100 \%$

Fuente: Elaboración propia

Del grafico anterior, se observa que el $100 \%$ de los pobladores están totalmente de acuerdo con que el Derecho a la Salud y el Medio Ambiente son derechos que tienen una gran relación entre sí.

7. ¿Considera que las acciones que toma el Estado respecto a la Contaminación Ambiental por plomo es la correcta?

Del $100 \%$ de los encuestados, el 80\% opina que el Estado no toma las adecuadas acciones respecto a la Contaminación Ambiental por plomo, considerándola ineficaz y el otro $20 \%$ no está de acuerdo con respecto a las acciones que toma el Estado concerniente a la Contaminación Ambiental.

\section{PREGUNTA $\mathbf{N}^{\circ} 7$}

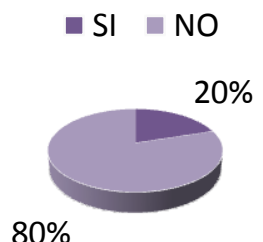

Fuente: Elaboración propia

Del grafico anterior, podemos observar que el $80 \%$ de los encuestados considera que el las acciones tomadas por el Estado 
respecto a la contaminación ambiental por plomo no son las correctas.

8. ¿Cree usted que se debería tener más control a las Empresas Metalúrgicas que contaminan el ambiente en donde vive?

Del $100 \%$ de los encuestados, el $100 \%$ opina que si se debería de crear formas de control más efectiva para controlar la contaminación que producen las empresas metalúrgicas de la zona y mejorar la calidad de vida de todos.

\section{PREGUNTA N 8}

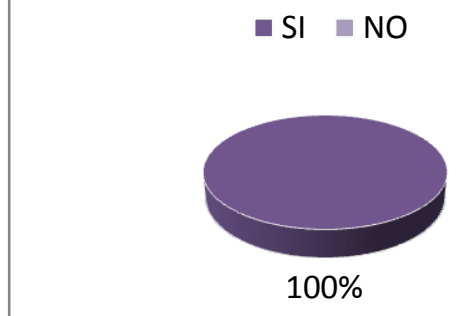

Fuente: Elaboración propia

Del grafico anterior, podemos observar que el $100 \%$ de los encuestados cree que si se debería tener más control sobre la contaminación del medio ambiente por parte de las empresas metalúrgicas.

9. ¿Considera usted que las personas más vulnerables y expuestos a estos arsénicos son los niños y los ancianos?

Del $100 \%$ de los encuestados, el 90\% opina que efectivamente los más vulnerables y expuestos a la contaminación de la zona son los niños y ancianos causándoles serios problemas a su salud y el otro $10 \%$ opina que no son los más vulnerables.

\section{PREGUNTA $\mathbf{N}^{\circ} \mathbf{9}$}

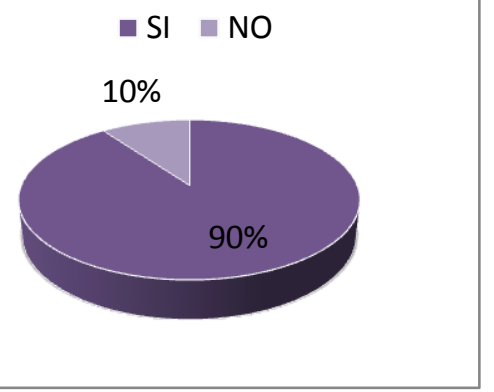

Fuente: Elaboración propia

Del cuando anterior, podemos observar que el $90 \%$ de los encuestados, determino que los niños y los ancianos son los más vulnerables y propicios a contaminarse y sufrir de alguna enfermedad o mal formación.

TABLA N ${ }^{\circ} 10$ : Total de respuestas de los encuestados.

\begin{tabular}{rrr}
\hline & SI & NO \\
\hline 4 & 1 & 9 \\
5 & 6 & 4 \\
6 & 10 & \\
7 & 2 & 8 \\
8 & 10 & \\
9 & 9 & 1 \\
\hline
\end{tabular}

Fuente: Elaboración propia.

IV.-

DISCUSIÓN

\section{YCONCLUSIONES}

En el presente artículo sobre la discusión se establece la interpretación de los resultados obtenidos y recolectados a partir de la problemática de investigación por lo que es importante resaltar que no es la repetición de los resultados, por lo contrario es la redacción y juicios de valores que le da sentido a los resultados. 
Antes de exponer la discusión del análisis que hemos recogido de los entrevistados, cabe indicar previamente que los entrevistados (expertos en materia de derecho constitucional y ambiental a la vez expertos médicos) han tenido más punto de coincidencia y convergencia que divergencias. Todos coinciden en señalar que el derecho a un ambiente sano y el derecho a la salud son derechos fundamentales que requieren una atención especializada y comprometida por parte de las entidades encargadas asimismo de la población. Los entrevistados aducen que si bien hace falta maquinaria, herramientas, logística y personal idóneo y capacitado para hacer respetar el derecho a la salud y a un ambiente sano, hace falta - por sobre todo- decisión política y compromiso medioambiental de las empresas y capacitación de parte de los pobladores. Los entrevistados me señalaron que es un problema que puede ser mejor manejado si los actores involucrados ponen de su parte.

A continuación vamos a detallar la discusión del análisis de los resultados de la entrevista.

De acuerdo a los resultados del trabajo de campo contrastado con los antecedentes internacionales y los antecedentes nacionales, y todo lo desarrollado en el presente trabajo de investigación, se ha analizado cómo pese a existir normativa aún se vulnere el Derecho a la Salud por Contaminación Ambiental por plomo en el Distrito de Ventanilla 2017, se ha obtenido los siguientes resultados:

a) En base a las entrevistas, se advierte que pese a existir normativa aún se vulnera el Derecho a la Salud por Contaminación Ambiental por plomo, debido a que no existe un adecuado control a cargo de los órganos fiscalizadores que deberían de proteger y velar por nuestros derechos, que en base a ello los habitantes del Distrito de Ventanilla se les vulnera su Derecho a la Salud al ser expuestos a terribles excesivas cantidades de Contaminación por plomo causadas por las empresas metalúrgicas y el no control por parte de los entes fiscalizadores que son regulados para la protección del medio ambiente, vulnerando así su derecho a la vida, la salud, medio ambiente conforme lo detallan los artículos $2^{\circ}$ inciso 22 y $7^{\circ}$ de la Constitución Política del Perú, donde se confiere una adecuada protección al derecho a la salud $\mathrm{y}$ ambiente, siendo que se considera que la forma de prevenir la Contaminación Ambiental por plomo en el Distrito de Ventanilla También los entrevistados han señalado de modo convergente que existe una serie de deficiencia, limitaciones y carencia que la Municipalidad Distrital deberá asumir y resolver.

b) Asimismo, es corroborado por el análisis documental, con la sentencia del Expediente $\mathrm{N}^{\circ}$ 009-2017-OEFA/TFA-SEPIM , proceso interpuesto por $\mathrm{La}$ Dirección de Supervisión Ambiental sobre la empresa Metalexacto S.R.L, respectivamente dentro de los considerandos y fundamentos expuestos en la referida se fundamenta la vulneración del 
Derecho a la Salud por Contaminación Ambiental por plomo en el Distrito de Ventanilla, aduciendo que la falta de control ambiental es el problema central en cuanto a fiscalización, por lo siguiente que señala "La cuestión en el presente caso versa sobre el dictado de una medida preventiva por parte de la Dirección de Supervisión consistente en el cese inmediato de toda forma de emisiones producto de sus actividades en la Planta Ventanilla mientras no acredite ante la Dirección de Supervisión la actualización del instrumento de gestión ambiental aprobado por la autoridad competente conforme a Ley", siendo así el resultado que se obtuvo de acuerdo al mencionado objetivo general.

c) De igual forma, se ratifica por el cuestionario, donde el 100\% aseguran que si existe la vulneración del Derecho a la salud por Contaminación Ambiental por plomo en el distrito de Ventanilla pese a existir normativa que la regule, declarando que el $60 \%$ de las personas que realizaron el cuestionario no conocen de centros de salud donde brinden información sobre contaminación ambiental en el Distrito de Ventanilla, y a pesar de ello, el $80 \%$ conoce los síntomas y daños que produce en el cuerpo la contaminación por plomo ya que son perjudicadas por la terrible contaminación por plomo a diario ocasionando problemas en su salud.

d) Al respecto, los resultados del objetivo específico 1; es determinar cómo se relacionan las normativas ambientales con el Derecho a la salud. Cuyo supuesto jurídico es que los Derechos fundamentales estipulados dentro de nuestra Constitución ya que están relacionados con las normativas ambientales para el bienestar de todos dentro del cual se encuentran desarrollados apegados a la normativa y la realidad el Derecho a la Vida y el Derecho a un Medio Ambiente sano, en base a los siguientes instrumentos:

Las entrevistas realizadas a los especialistas en derecho constitucional, derecho ambiental y la rama de la medicina, que refieren a la relación de las normativas ambientales con el Derecho a la Salud, otorgándole que si existe una relación entre normativa y la realidad aplicada al Distrito de Ventanilla, donde la problemática versa en tener un ambiente sano y como consecuencia afectaría nuestra salud según las normativas vigentes, ya que nuestra Constitución Política del Perú las vincula de manera doctrinal y aplicada a la realidad, del cual no se cumple con el Derecho a la Salud conforme lo señala la Constitución por falta de control y fiscalización por parte de la Municipalidad Distrital de Ventanilla y en todo caso de la Región Callao con respecto a la Contaminación Ambiental por plomo en el distrito siendo estos regulados en la Ley General de la Salud $\mathrm{N}^{\circ} 26842^{\circ}$; estos derechos son establecidos como fundamentales porque son primordiales para llevar una vida con dignidad teniendo en cuenta que es el primer artículo de la 
constitución y el más esencial; tener un desarrollo pleno y con bienestar en la sociedad siendo este mismo cargo del estado, tanto en al ambiente como en la persona.

En ese sentido, de acuerdo a los resultados obtenido en la presente investigación resulta ser igual a la igual tesis nacional sustentada por Pacheco, M (2014) cuyo título es "Conciencia Ecológica: Garantía de un Medioambiente Sano" de la Universidad Pontificia Universidad Católica del Perú. En una de sus conclusiones señala que el impulso de la conciencia ecológica favorece a la población bien informada y educada sobre la necesidad del desarrollo sostenible, para que exija la formulación de políticas de desarrollo que traten el crecimiento económico y la conservación ambiental.

Tras lo mencionado en el párrafo precedente y en comparación con los resultados obtenidos mediante lo instrumentos, corroboramos identificar como la Contaminación Ambiental por plomo de las empresas metalúrgicas del Distrito de Ventanilla perjudican el bienestar de los pobladores principalmente a niños y ancianos, ahora es oportuno mencionar que las empresas metalúrgicas en relación a la contaminación ambiental referido por el Tribunal Constitucional expone la importancia y relevancia que tiene la salud y el ambiente con una problemática real como es la contaminación, ya que con ello se comprueba que los estándares de calidad dañados por la Contaminación Ambiental por plomo es causal de preocupación y atención conjuntamente con el accionar de las autoridades competentes que no toman las medidas adecuadas para dejar de contaminar y de controlar la contaminación ya encontrada, con ello el Tribunal Constitucional como en el caso presentado llego a términos donde no solo se basaban en legislación nacional sino interpretaciones internacionales que avalan nuestros actos y el hecho de contaminación por plomo presente en nuestro país.

e) Por consecuente, nuestro supuesto jurídico específico 2 menciona la causa de la Contaminación Ambiental en el Distrito de Ventanilla es la falta de control que no tiene el Distrito con aquellas empresas metalúrgicas que trabajan en la zona; de modo que, las mismas funcionan sin cumplir con las medidas necesarias para el uso y elaboración ello afecta la salud en el Distrito y que ha llegado a niveles muy altos en los últimos años afectando a la población.

Como lo respalda la Ley General del Ambiente $\mathrm{N}^{\circ}$ 28611, bajo esta cita, se tiene un especial reconocimiento también en temas ambientales aduciendo que en materia ambiental las personas tienen el derecho de saber cuáles son las obras, medidas, políticas y normas aplicadas a su distrito y sobre la explotación de los recursos naturales del lugar en donde habitan que son derecho de todos, poniendo en acuerdo con los resultados en la investigación aclarando el tanto la ley como los resultados coindicen en las respuestas obtenidas sobre la evidente Contaminación Ambiental por plomo encontrada en el Distrito de Ventanilla y la conservación de los recursos naturales al igual que la salud de los pobladores entre los 
más vulnerables niños y ancianos. Habiendo realizado un análisis de los resultados de los instrumentos, en los cuales hemos recabado información importante que sustenta nuestra investigación en virtud a que en la citada sentencia el Tribunal Constitucional, se puede explicar que la vulneración y afectación que causa el plomo en la población es un daño irreparable, por lo que se demostró como la Contaminación Ambiental por plomo de las empresas metalúrgicas del Distrito de Ventanilla perjudican el bienestar de los pobladores principalmente a niños y ancianos, producto de las empresas metalúrgicas que almacenan, conservan, procesan y transforman estos metales a otros elementos que puede ser empleados para el uso diario, como el producción de baterías tal y como lo menciona el informe realizado por la OEFA mencionado en líneas anteriores los índices sanitarios del lugar están muy por debajo del mínimo que debe de tener para poder llegar una vida sana, a sabiendas que el plomo es el agente contaminante con más razón aún se viene estableciendo parámetros en cuanto a contaminación por plomo y los daños que atraviesan en el ambiente todos los ciudadanos del Distrito de Ventanilla.

\section{Discusión sobre los resultados del análisis de la encuesta poblacional}

La encuesta poblacional aplicada en el presente estudio ha logrado su cometido pues nos permitió contrastar los supuestos jurídicos formulados. Además fue interesante saber de modo directo la versión, posición, percepción y opinión de los propios pobladores. Por ello y a fin de que se cuente con una muestra significativa fue aplicada a 10 pobladores del distrito de Ventanilla.

También para la encuesta se tuvo en cuenta la opinión de jóvenes, adultos, varones y mujeres y de toda condición social, cultural y económica. Con ello la muestra de la encuesta cobra relevancia pues se trata de una muestra aplicada a un grupo poblacional diverso y que expresa el sentir y la percepción de la población en general respecto a la problemática.

Resultó necesario también aplicar la encuesta para que la información obtenida de la fuente documental y de las entrevistas, se contrastara y se obtengan resultados los más objetivos y certeros posibles. En ese sentido se formularon preguntas que estaban directamente relacionadas con los objetivos de la investigación.

Respecto a las Conclusiones que plantea el artículo, estos son tres y se detallan a continuación. Primera conclusión:

A partir de los Resultados obtenidos y de la Discusión planteada para el presente estudio se ha analizado quedando demostrada la vulneración del Derecho a la Salud por Contaminación Ambiental por plomo en el Distrito de Ventanilla 2017, sustentado en base a las entrevistas, análisis documental y cuestionario, instrumentos que ayudaron a corroborar la presente investigación, dicho en el artículo $2^{\circ}$ inciso 22 y el artículo $7^{\circ}$ de la Constitución Política del Perú con relación a la Ley General de Salud $26842^{\circ}$ artículo $103^{\circ}$ y la Ley General del Ambiente $28611^{\circ}$ en su artículo $31^{\circ}$, respetando el criterio y análisis adoptado ante esta problemática, comprendiendo 
que estos derechos fundamentales son primordiales para toda persona y para el bienestar de una sociedad.

Segunda conclusión:

Se concluye, que se ha determinado la relación de las normativas ambientales con el Derecho a la Salud en base a las entrevistas, análisis documental, cuestionario, instrumentos que ayudaron a corroborar la presente investigación donde considera al Derecho a la Salud como una garantía indispensable para el goce de los derechos económicos y sociales, en consecuencia dando énfasis a la salud y calidad ambiental conforme se detalla en la discusión, quedando demostrado adecuadamente la interrelación de la normativa ambiental con el Derecho a la salud siendo de exigencia obligatoria para personas y entidades públicas o privadas.

Tercera conclusión:

Se concluye que se ha identificado que la Contaminación Ambiental por plomo de las empresas metalúrgicas del Distrito de Ventanilla perjudican el bienestar de los pobladores principalmente a niños y ancianos en base a las entrevistas, análisis documental, cuestionario instrumentos que ayudaron a corroborar la presente investigación como la conforme se detalla en la discusión, por lo tanto queda demostrado el daño ocasionado por Contaminación Ambiental por plomo regulado en la Ley General de la Salud $26842^{\circ}$ en su artículo $103^{\circ}$, estableciendo la existencia de estándares de calidad y responsabilidad con el medioambiente en cuanto agentes contaminantes.

Tal como se señaló al inicio del presente artículo se plantean además las siguientes

recomendaciones:

Primera recomendación:

En relación con la Discusión de Resultados, se recomienda, que no se siga vulnerando el derecho a la salud en el Distrito de Ventanilla disminuyendo la contaminación ambiental por plomo en el Distrito realizando una mayor fiscalización en cuanto al medio ambiente para que garanticen, protejan, y salvaguarden la calidad de vida de los ciudadanos dicho en el artículo $2^{\circ}$ inciso 22 y el artículo $7^{\circ}$ de la Constitución Política del Perú con relación a la Ley General de Salud $26842^{\circ}$ artículo $103^{\circ}$ y la Ley General del Ambiente $28611^{\circ}$ en su artículo $31^{\circ}$, respetando el criterio y análisis comprendiendo que estos derechos fundamentales son primordiales para toda persona y para el bienestar de una sociedad.

Segunda recomendación:

En relación a los objetivos, la creación de campañas de salud dando a conocer los problemas presentes en cuanto al medio ambiente que los rodea, los conocimientos básicos en cuanto a sanidad y calidad de vivir en un medio ambiente sano y limpio a los pobladores del distrito de ventanilla a la par de la concientización medioambiental respecto a lo importante que es vivir en un entorno limpio y equilibrado, relacionando la normativa ambiental con el derecho a tener buena salud por lo que se haría que una población educada y con formación haga respetar sus propios derechos para el bienestar de todos. 
Tener un plan de prevención para que las empresas metalúrgicas dejen de emitir cantidades desmesúrales de plomo sin control alguno al ambiente poniendo en riesgo el bienestar principalmente de los niños y ancianos del Distrito de Ventanilla, y al mismo tiempo busquen depurar todas aquellas maquinarias toxicas de la zona que siguen presentes y siguen contaminando por suelo, agua y aire, ya que en base a este plan de prevención de la contaminación por plomo tendría que ser respetado por toda persona natural o jurídica se podría evitar poner en riesgo las vidas de los niños, ancianos y de todos los pobladores del lugar que ya se encuentran afectadas por el plomo presente en su entorno.

Con las recomendaciones planteadas creemos el Distrito de Ventanilla podrá afrontar de modo más adecuado y acorde a la normativa que reconoce el derecho a la salud y a un medio ambiente sano.

\section{Referencias:}

Aldana M. (2012). Normativa en Salud Ambiental y Empresa. Universidad de Lima: Fondo Editorial.

Acosta, A. (2003). El Desarrollo como asignatura Global, Nacional y Local. Ecología, Economía y Ética del Desarrollo Sostenible. Quito: Abya Yala

Agencia de Protección Ambiental, (2017). Qué se puede hacer para proteger a la familia contra la intoxicación por plomo. Recuperado de: http://extwprlegs1.fao.org/docs/pdf /per161555anx.pdf

Alva, K. (2014). Fortalecimiento de la protección al ambiente y los recursos naturales en la Constitución Peruana de 1993. (Tesis de Abogada, Universidad Privada Antenor Orrego). (Acceso el 21 de Setiembre del 2017)

Comité de Derechos Económicos, Sociales y Culturales. (2015). Observaciones finales sobre el tercer informe periódico. Recuperado de: http://tbinternet.ohchr.org/Treaties/ CESCR/Shared\%20Documents/H ND/INT_CESCR_CSS_HND_240 64 S.pdf

Comisión Europea. (2015). Plan de Acción sobre la Eco innovación. Francia. Recuperado de

https://ec.europa.eu/environment/e coap/about-eco-innovation/goodpractices/france/505 es

Comisión Interamericana de Derechos Humanos. (1983). El Sistema Interamericano de Protección De Los Derechos Humanos. Recuperado de: file:///C:/Users/Usuario/Download s/Sistema Interamericano de Der echos_Humanos._Versi_n_actuali zada_para_este_curso._Centro_de Derechos Humanos 2007..pdf

Comisión Interamericana De Derechos Humanos. (1985). Anuario Interamericano de Derechos Humanos, Washington D.C. Recuperado de: http://www.corteidh.or.cr/docs/opi niones/seriea_05_esp.pdf

Comisión Interamericana De Derechos Humanos. (1997). Informe sobre la situación de los derechos humanos en Ecuador. Recuperado de: http://www.cidh.org/countryrep/Ec uador-sp/Capitulo\%209.htm

Comisión Interamericana De

Derechos Humanos. (1997). 
Informe sobre la admisibilidad de la Comunidad de San Mateo de Huanchor de Perú. Fundamento 49. Recuperado de: https://www.cidh.oas.org/annualre p/2004sp/Peru.504.03.htm

Comité de Derechos Económicos, Sociales y Culturales. (2013). "Observación general $N^{o} 14$, sobre el derecho del niño a que su interés superior sea una consideración primordial". Recuperado de: https://confdts1.unog.ch/1\%20SPA/Tradutek/ Derechos_hum_Base/CESCR/00 1 obs grales Cte\%20Dchos\%20E c\%20Soc\%20Cult.html

Declaración de Río. (1992). Conferencia De Las Naciones Unidas Sobre El Medio Ambiente Y El Desarrollo. Recuperado de: http://www.cedaf.org.do/eventos/f orestal/legislacion/inst internac/de claracion_rio.pdf

Ely, A. (2006). Derechos económicos, sociales y culturales en América Latina. México: Plaza y Valdéz S.A.

Guiza, L. (2016). Actualidad y desafíos del derecho minero colombiano. Bogotá: Universidad del Rosario.

Gutiérrez, C. (2006). El derecho al medio ambiente adecuado como derecho humano. Colombia: Centro Editorial Rosarista.

Hernandez, A. (2000). El cuidado del Medio Ambiente. Mexico: Universidad Autonoma del Estado de México.

Huayhua, L. (2013). La Respuesta Estatal para solucionar el problema de Contaminación Ambiental por Plomo en el callao y sus efectos en la protección del derecho a gozar de un ambiente equilibrado y adecuado al desarrollo de la vida y a la salud de la población afectada. (Tesis de Magister, Universidad Católica del Perú). (Acceso 22 de Setiembre).

Knox, J. (2016). Obligaciones de Derechos Humanos relacionadas con el disfrute de un medio ambiente sin riesgos, limpio, saludable y sostenible. Recuperado de:https://documentsddsny.un.org/ doc/UNDOC/GEN/G16/015/75/P DF/G1601575.pdf?OpenElement

Ley General del Ambiente. (2005). Ministerio del Ambiente. Recuperado de: http://www.minam.gob.pe/wpcontent/uploads/2013/06/leygeneral-del-ambiente.pdf Maneiro, Y. (2007). La tutela de los derechos fundamentales $y$ libertades públicas por los tribunales laborales. España: Gesbiblo S.L

Organización Mundial de la Salud. (2017). Intoxicación por plomo y salud. Recuperado de: http://www.who.int/mediacentre/fa ctsheets/fs379/es/

Organización Panamericana de la Salud. (1986). Centro Panamericano de Ecología Humana y Salud, Organización Mundial de la Salud. Recuperado de:

http://www.bvsde.paho.org/bvsacd leco/006019.pdf

Pacheco, M. (2014). Conciencia Ecológica: Garantía de un Medioambiente Sano (tesis para el Título de Magister, Pontificia Universidad Católica del Perú). (Acceso 21 de Setiembre del 2017). 
Pérez, M. (2010). Cinco miradas sobre el derecho a la salud. México: Fundar Centro de Investigación A.C.

Primera Cumbre de las Américas. (2015). Prosperidad con Equidad: El Desafío De La Cooperación En Las Américas. Recuperado de:http://www.oas.org/36ag/espan ol/doc referencia/cumbreamericas miami_planaccion.pdf

Ramírez, S. (2016). Cuando la enfermedad se silencia: Sida y toxicidad en el Oriente Boliviano. Tarragona: Publicacions URV.

Sanchez, H, (2001). Métodos e indicadores para la evaluación de los servicios de salud. Espeña: Universidad Autonoma de Barcelona

Sentencia del Tribunal Constitucional (Octubre, 2004), en el expediente 2016-2004-AA/TC sobre Acción de Amparo interpuesto por Don José Luis Correa Condori. Fundamento 26. Recuperado de: http://tc.gob.pe/jurisprudencia/200 5/02016-2004-AA.pdf

Sentencia del Tribunal Constitucional (Abril, 2004), en el expediente 2480-2008-AP/TC sobre Proceso de Amparo interpuesto por Don Ramón Medina Villafuerte. Fundamento $\mathrm{N}^{\circ} \quad 6 . \quad$ Lima: Tribunal Constitucional del Perú, Centro de Estudios Constitucionales.

Sétima Cumbre de las Américas. (2015), Prosperidad con Equidad: El Desafio De La Cooperación En Las Américas. Recuperado de: http://www.summitamericas.org/vii/docs/mandates_es .pdf
Valencia, A. (2011). Salud e igualdad sustantiva: repesando el derecho a la salud desde el enfoque de capacidades humanas en el Derecho frente a la pobreza: los desafíos éticos del constitucionalismo de los derechos. Lima: Palestra.

Villarán, L. (2016). Constitución Política del Perú comentada. Perú: Tribunal Constitucional del Perú, Centro de Estudios Constitucionales.

\section{Referencias Metodológicas}

Alarcón, R. (2013) Métodos y diseños de investigación del comportamiento. Lima:

Universidad Ricardo Palma.

Albert Gómez, María José (2007), La investigación educativa. Claves teóricas.

Madrid: McGraw Hill.

Bermúdez, L. (2016). Investigación en la gestión empresarial. España: Ecoe Ediciones.

Bernal, César A. (2006) Metodología de la investigación, segunda edición. México:

Pearson.

Blaxter, L., Hughjes, Ch., Tight, M. (2008) Cómo se investiga. Barcelona. Graó.

Briones, Guillermo (1998) Métodos y técnicas de investigación para las Ciencias Sociales. México. Trillas,

Bunge, Mario (1969) La investigación científica. Su estrategia y su filosofía. Bs As.: Ariel.

Castelló, M. (2007) Escribir y comunicarse en contextos 
científicos y académicos.

Barcelona: Graó.

Chavaría, M.; Villalobos, M. (2000) Orientaciones para la elaboración y presentación de tesis. México: Trillas.

Delgado, J. M., Gutiérrez, J. (1999) Métodos y técnicas de investigación en Ciencias

Sociales. Madrid: Síntesis.

Díaz, V. (2009). Metodología de la investigación científica y bioestadística. Chile: Ril.

Eco, U. (1998) Cómo se hace una tesis: técnicas y procedimientos de investigación,

Estudio y escritura. Barcelona: Gedisa.

Flick, U. (2004) Introducción a la investigación cualitativa. Madrid: Ediciones Morata.

Habermas, J. (1988) La lógica de las Ciencias Sociales. Madrid: Tecnos.

Hernández Sampieri y otros (2003) Metodología de la Investigación. México: Mc Graw

Hill Interamericana.

Huamanchumo Venegas Henry y Rodríguez Figueroa Jorge (2015), Metodología de la Investigación en las Organizaciones, Lima Perú 1ra.Edicion Editorial Summy.

Ibáñez Brambilla, B. (1999) Manual para la elaboración de tesis. México: Trillas.

Méndez A., Carlos E. (2001) Metodología. Diseño y desarrollo del proceso de Investigación, tercera edición. Bogotá: McGraw Hill,
Namakforoosh, M. N. (2002) Metodología de la Investigación. México: Limusa.

Pardinas, Felipe. (1969, 1999) Metodología y Técnicas de investigación en Ciencias

Sociales. México: Siglo XXI Editores.

Popper, K. R. (2008) La lógica de la investigación científica. Segunda edición. Madrid: Tecnos.

Polit. D., Hungler, B. (1997) Investigación Científica en Ciencias de la Salud. Quinta Edición. México: Mcgraw Hill Interamericana.

Reguera, A. (2008). Métodos cualitativos y cuantitativos en investigación evaluativa". Madrid: Ediciones Morata. 